\title{
Fracture Energy of Materials Exposed to Pulse Action
}

\author{
Krivosheev S. I. , Magazinov S. G. \\ Peter the Great Saint-Petersburg Polytechnic University, Russia
}

Copyright $(2016$ by authors, all rights reserved. Authors agree that this article remains permanently open access under the terms of the Creative Commons Attribution License 4.0 International License

\begin{abstract}
To study the process of brittle fracture, initiated by a microsecond range pulse action, we applied the magnetic pulse technique of controllable pressure pulse generation with pulse amplitude up to $2 \mathrm{GPa}$. The threshold energy level has been found, and related to a parameter specific of each material - energy accumulation time. With application of the thermodynamic approach, we develop and demonstrate the destruction criterion that allows us to define this material parameter. Following the results of experimental data and numerical simulation, we show how to describe the destruction process both for samples with crack-type macro defects, and defect-free samples. The study reveals the dependency of the surface energy from the load build-up and material properties when exposed to magnetic pulse action.
\end{abstract}

Keywords Crack, Magnetic Pulse Method, Surface Fracture Energy, Pulse Strength, Shock Action

\section{Introduction}

The loss of strength properties and bearing capacity of samples is caused by de-homogenization of material structure and occurrence of structural integrity rupture zones. Multiple papers examine the issues of fracturing and fracture propagation under various loading conditions. However, an adequate analysis of brittle fracture under conditions of pulse loading has not been definitely shaped yet. The destruction process analysis is a challenge due to the influence of multiple factors and singularities, related to material properties, exposure parameters, variety and structural difference of force fields during the experiments.

The aim of the present study is to demonstrate the possibility of the magnetic-pulse technique for investigation of the process of pulse material fracture and clarify some general details of the generation of new surfaces at material fracture under the high-rate loading.

Tests, carried out with various materials, exhibit the threshold nature of fracture and increase of failure amplitude in response to loading pulse contraction during destruction of both defect-free samples, and those having crack-type macro-defects [1-3]. Seen below are the results of experimental research and analysis of material destruction when exposed to magnetic pulse action.

\section{Distinctive Features of Magnetic Pulse Action}

The multitude of sample stress states and loading conditions can be significantly limited by defining pulse loading modes with parameters, listed below.

Variants of loading schemes for pulse action exposure:

- Stress field is formed, its spatial localization is substantially smaller than sample sizes (magnetic pulse shock action);

- Loading is conducted under known and controllable in process of loading formation parameters;

- The sample can be considered as an energy-closed system, for when load pulse action expires, there is no energy interchange between the sample and the loading device.

Such loading modes are performed at laboratory level when studying material destruction upon spallation loading schemes [3-6]. In addition to spallation loading schemes, the magnetic pulse technique also enables to conduct pulse loading with the listed above parameters while studying the destruction of samples with crack-type macro-defects [7, 8]. This is valid for a number of applied technologies in the sphere of finely ground material production and ore mineral concentration, as well as in the sphere of natural and man-induced earthquakes [9].

\section{Magnetic Pulse Technique Used to Create Controllable Pressure Pulse}

It has been known, that when the current flows on closely spaced conductive parts, the latter are subjected to the action of mechanical force, caused by interaction of the flowing current and magnetic field created by this current. In general case, the direction and distribution of acting forces is specified by current distribution on cross-section of conductive parts, depending on their geometric sizes and 
relative alignment. In case of perfect conductivity, a normal force (magnetic pressure) acts on conductor's surface unit:

$$
\bar{P}_{m}=\frac{1}{2} \cdot[\bar{J}, \bar{B}]
$$

where $\bar{J}, \bar{B}$ - surface current density and magnetic field density at a point on surface.

As it is described in [10], for flat parallel buses, divided by gap $\mathrm{h}$, that is substantially less than their width $\mathrm{b}$, one can neglect the fringe effect and consider the current $I(t)$ and magnetic field distribution as edgewise uniform. Under this estimation the instantaneous value of magnetic pressure $P_{m}(t)$, acting on buses from the side of gap, can be determined as:

$$
P_{m}(t)=\frac{1}{2} \cdot \frac{I(t)}{b} \cdot B(t)=\frac{1}{2} \cdot H(t) \cdot \mu H(t)=\frac{B(t)^{2}}{2 \cdot \mu}
$$

where $B(t)$ и $H(t)$ - inductance and strengths of magnetic field accordingly, $\mu=\mu_{0}=4 \cdot \pi \cdot 10^{-7} \mathrm{H} / \mathrm{m}$.

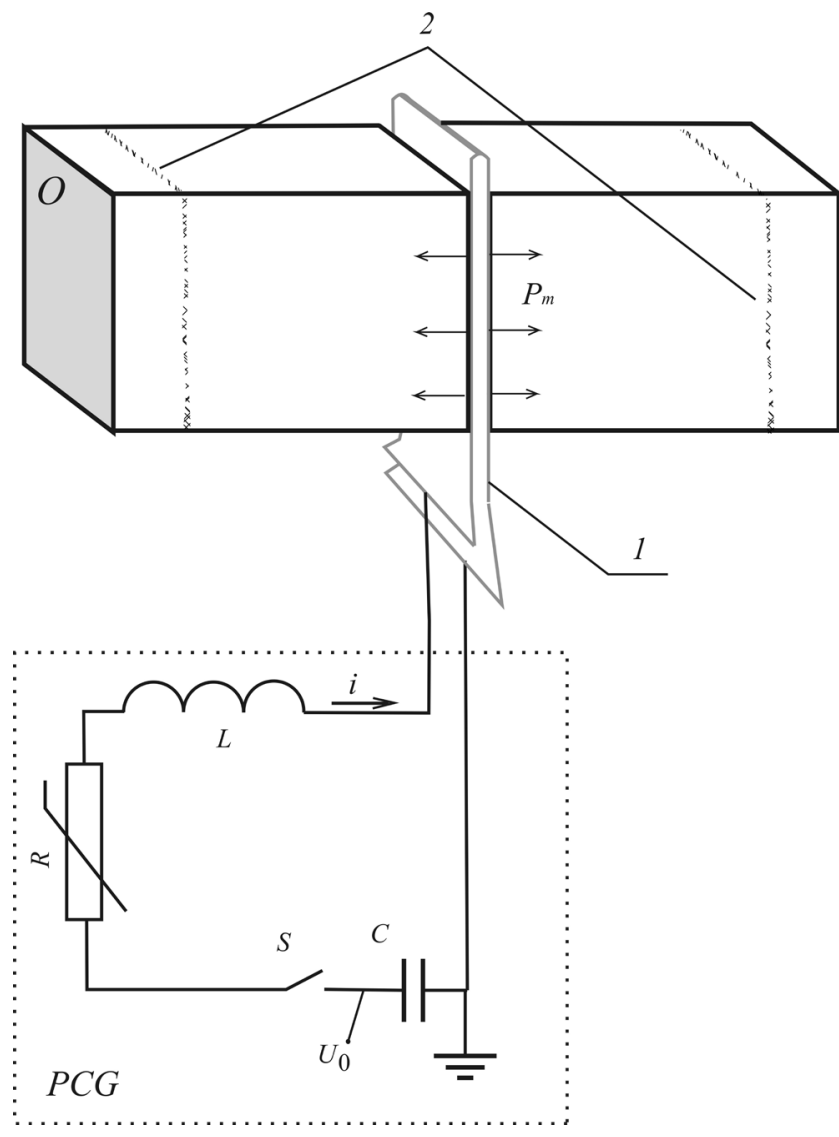

Figure 1. Schematic circuit of pulse current generator (PCG) device and sample schemes of loading for pulse testing of spalling $C, L$ - capacitor and self-inductance of PCG; $U_{0}$-voltage: $S$ - high-voltage commutation switch; $R$ - nonlinear resistor; $O$ - sample under test. 1 - loading device, 2 - spall areas. Sizes are in $\mathrm{mm}$.

In the event of uniform current distribution on buses as wide as $b$, the values of pressure pulse, acting on them, are uniquely related to the values of current pulse and expressed by equation (2). In such a case a complicated problem of pressure pulse measurement is reduced to a simple procedure of current pulse measurement [8].

Pulse current generator (PCG) circuit with flat buses for pulse testing of spalling can be seen in Fig.1.

A generator PCG-125, equipped with two pulse low-inductance KMK-50-6 type capacitors of $6 \mu \mathrm{F}$ each, charging voltage up to $50 \mathrm{kV}$ and acting as storage batteries, has been used to carry out the experiments. This generator allows getting a unidirectional pulse under pulse loading up to $20 \mathrm{nH}$, its amplitude is up to $600 \mathrm{kA}$ of 4 microseconds, that corresponds to the pressure pulse of up to $2000 \mathrm{MPa}$.

The technique used to control the current pulse form as well as the requirements to parameters of the loading device are given in [8].

\section{Destruction of Samples with Crack-type Macro-defects}

Fracture of samples, made of various materials, was studied, following the magnetic pulse loading technique, described in [8]. Samples with typical sizes, given in Fig.1, were used for these experiments. By exposing such samples to loading at micro-second loading duration, the magnetic pulse shock conditions are formed, as opposed to experimental conditions, described in $[11,12]$. Due to the symmetric nature of loading and sample symmetry, propagation of the main crack (MC) is nearly unidirectional [1].

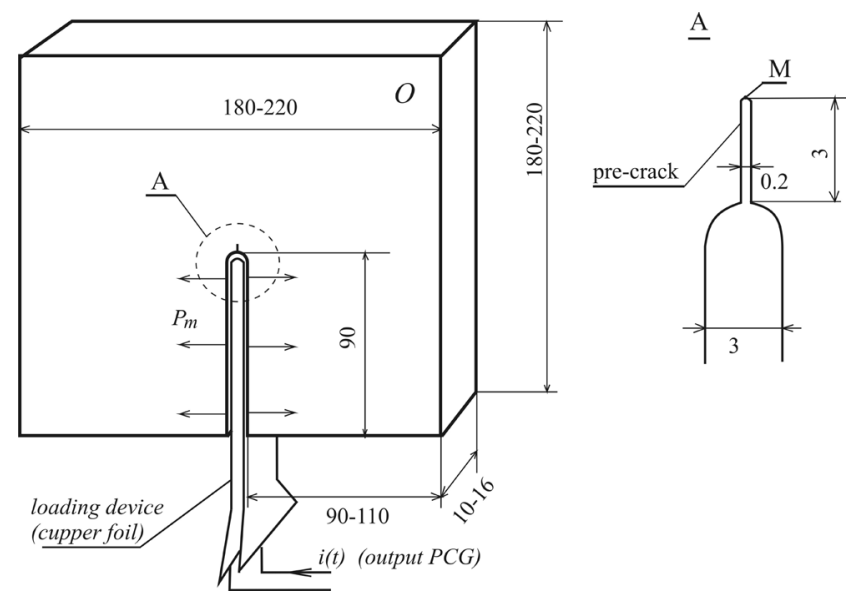

Figure 2. Test sample $O$ with a loading device, PCG generator outlet connectable.

Shown in Fig. 3 - typical dependences of the propagated crack length in samples with crack-type macro-defects, loaded along the macro fracture zones in accordance with mode I by uniformly applied pulse pressure, on pulse amplitude and loading duration. As illustrated by PMMA fracture (curves 7, 8, 9 in Fig.3), one can see the growth of threshold fracture loads, concurrently with the exposure time decrease. Threshold fracture loads $P_{t r}$ can be specified by projecting the propagated crack length $L_{c r}$ into the area 
$L_{c r} \rightarrow 0$, as illustrated by curve 4 .

The dynamics of crack propagation has been studied with the use of PMMA (poly methyl methacrylate) optically-transparent samples, according to the method, presented in [14]. A super-speed camera (SFR-2), working in photochronograph mode, was used for image registration and recording. A movie fragment with the start of crack progression is shown in Fig. 4.

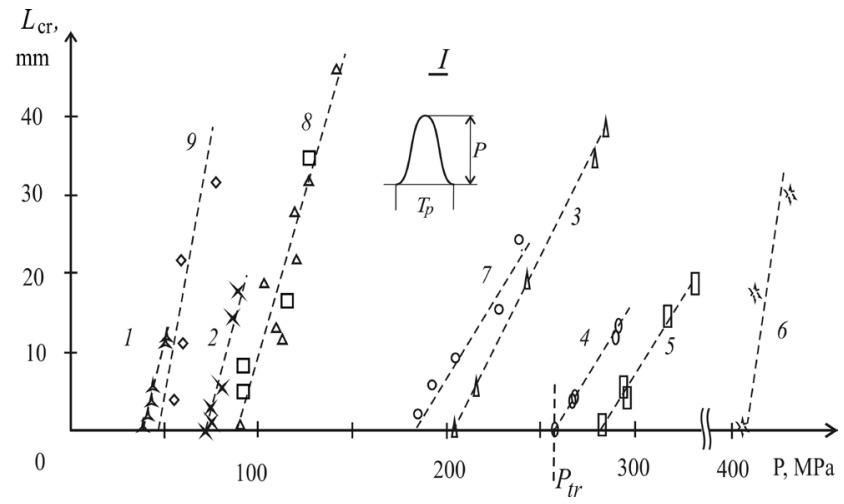

Figure 3. Dependences of the propagated crack length on the single loading pulse amplitude at loading duration: 1 - limestone, $4.4 \mu \mathrm{s} ; 2$ marble, $3.6 \mu \mathrm{s} ; 3$ - gabbro-diabase, $3.6 \mu \mathrm{s} ; 4$ - sandstone, $3.6 \mu \mathrm{s} ; 5$ granite, $3.6 \mu \mathrm{s} ; 6$ - polymer compound - $1.5 \mu \mathrm{s}$ [13]; PMMA: 7 - $2 \mu \mathrm{s} ; 8$ $4 \mu \mathrm{s} ; 9-8.6 \mu \mathrm{s}[7] . \boldsymbol{I}$ - pulse waveform.

Optical emission of electric discharge plasma, coupled out of the high-voltage triggered commutation switch into the registration zone (zone $R$ ), using optical fiber, makes it possible to synchronize the current pulse (pressure pulse) with the destruction (fracture) process. Like in case of the experiments, described above, there is a certain delay of main crack propagation $\mathrm{MC} \tau_{d}$ relative to the pressure pulse $P(t)$.

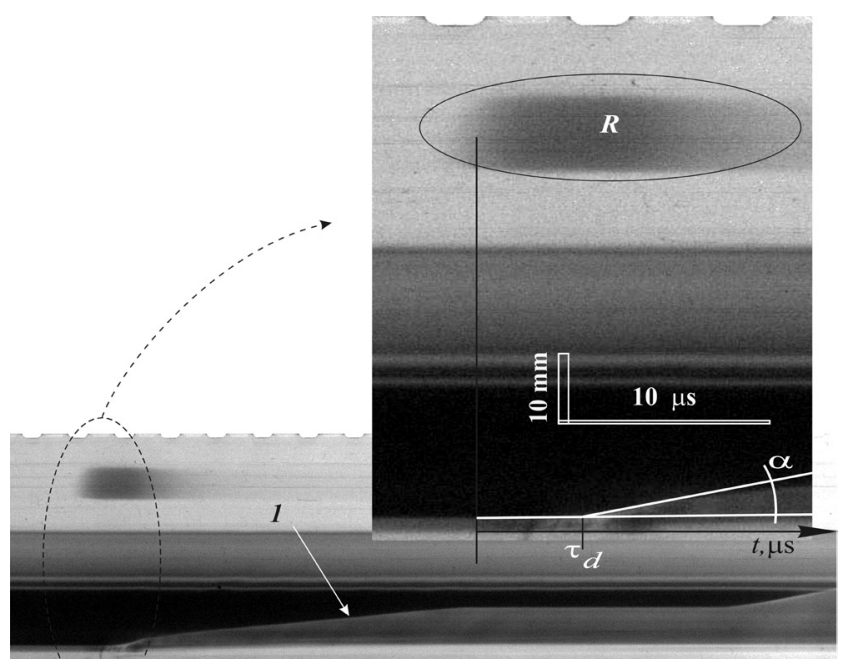

Figure 4. Movie fragment. 1 - crack apex path.

Starting crack propagation rate was determined from angle of inclination of tangential curve to the crack propagation path $\alpha$. The increase of the pulse amplitude by constancy of pulse duration increases the crack propagation rate at the initial phase. Further crack propagation behaviour is conditioned by loading waves, reflected from sample edges.

\section{Destruction Criterion}

The analysis of experimental data, related to crippling tests under mechanic action, can be reduced to detection of the stress-strain state of a sample and choosing a relevant fracture criterion.

In [15] are describing various types of criteria (force, energy, thermodynamic), used to estimate and define destruction processes under pulse loading. It should be expressly noted, that experimental and analytical research of pulse destruction was conducted by Russian Federal Nuclear Centre - All-Russian Scientific Research Institute of Experimental Physics. This research allows us to establish a time master dependency between the strength of different materials and thermodynamic invariant [16]

$$
J_{1}=P_{c r} /\left(G \rho\left(H+L_{m}\right)\right)
$$

where $P$ - critical density of absorbed energy, $G$ Gruneisen factor, $\rho$ - material density, $H$ and $L_{m}$ - enthalpy and melting heat and structural and time-dependent approach, proposed in [17].

It has been known, that the system enthalpy can be specified as:

$$
H=U+p V-\sum X_{i} y_{i}
$$

where $U$ - system internal energy, $X_{i}$ and $y_{i}$-integrated force and coordinate of non-mechanical force action. In the absence of non-mechanical forces, the system enthalpy is specified only by internal $U$ and potential energy $p V$. Assuming, that in the event of exposure to pulse action the process of potential energy accumulation is of adiabatic nature, that is to say, that it occurs at constant entropy $S \sim$ const , the enthalpy change will take the form $d H=V \cdot d p$, while internal energy change will depend only on the action of exterior forces and will be specified as $d U=p \cdot d V$. This explains the time dependence-strength correlation, described by Zhurkov model [18] with the use as internal energy parameter of materials and results of [16], obtained on basis of the thermodynamic approach. To evaluate the state of the system, let us introduce the ultimate (threshold) value of potential energy $p_{f r} V_{f r}$, which, when exceeded, brings the system into a destroyed (fractured) state.

Under dynamic (pulse) loading the potential energy is defined by time-dependent parameters $p(t)$ and $V(t)$. Given that the system transition from one state into another state takes time, we shall reckon, that the system transition into the destroyed state has been completed, once the potential energy achieves the ultimate value $p_{m} V_{m}$ within a certain time interval $\tau_{L}$. This statement can take the form 


$$
\frac{1}{\tau_{L}} \int_{0}^{t} p(t) V(t) d s \leq p_{m} V_{m}
$$

which, when violated, corresponds to system destruction.

The described situation is illustrated in Fig. 5. It is evident, that under such a scenario the destruction occurs when the volumes of a parallelepiped $V_{0} \cdot p_{0} \cdot \tau_{L}$ and of a hexahedron with vertices $A, A_{V, t}, A_{p, t}, t_{f r}, 0$ and faces, conditioned by the type of loading and strain dependency $0 A_{p, V}$ (example of linear dependency is seen in Fig. 5), are equal. In plane $p, t$ the curve $T D S$, setting apart the non-fractured $I$ and fractured $I I$ system states, is specified by parameters of a minimal failure loading with amplitude $A_{p, t}$ and duration $\cdot t_{f r}$.

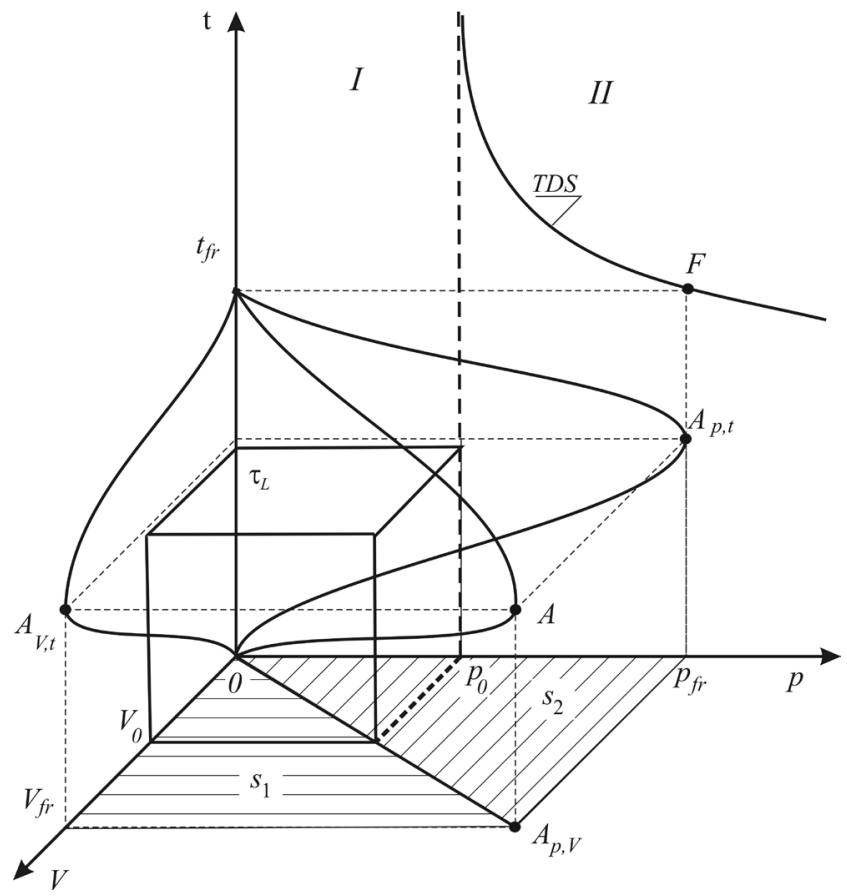

Figure 5. Destruction scenario

To describe the system behavior in conditions of one-dimensional elongation, implemented during material testing in accordance with spallation destruction pattern, the expression (5) can take the form:

$$
\frac{1}{\tau_{L}} \max _{x} \int_{0}^{t} \varepsilon(s, x) \sigma(s, x) d s \leq \varepsilon_{m} \sigma_{0}
$$

where $\varepsilon(s, x), \sigma(s, x)$ - actual values of strain and stress at a point with coordinate $\mathrm{x}, \varepsilon_{m}$-strain value, that corresponds to material breaking strength $\sigma_{0}$ under static conditions. Thus, assuming that the function $\varepsilon(s, x)$ is continuous from the start of the exposure until the moment, when the system transfers into the destroyed state, the expression (6) can take the form:

$$
\frac{1}{\tau_{L}} \varepsilon \xi \int_{0}^{t} \sigma(s, x) d s \leq \varepsilon_{m} \sigma_{0}
$$

here $\varepsilon \xi \in\left[\begin{array}{l}\min \varepsilon(t), \max \\ (0, t) \\ (0, t)\end{array}\right]$ depending on function type $\varepsilon(t)$, determined in the event of linear strain dependency exclusively by the parameters of loading pulse $p(t)$.

In this scenario the line $0 A_{p, V}$ in Fig. 5 represents the strain dependence $\sigma(\varepsilon)$ in the event of uniaxial material extension, and in general case is non-linear. For brittle materials this dependence is close to a linear one up until the destruction $[19,20]$, and the areas, conditioning the change of internal energy $S_{1}$ and enthalpy $S_{2}$, are equal.

In [21] we illustrate that the use of time-structural criterion $[7,17]$ for destruction process analysis is limited, as the criterion parameter depends on type of loading. The same principle of equal areas, used to describe the voltage-time characteristics of the gap electric strength [22, 23], proves that the form of incoming voltage significantly conditions the gap breakdown properties.

In [24] to describe fracture processes in metals, exposed to pulse loading, the chosen criterion, conceptually close to (6), is based on specification of the material threshold energy, released in critical area within a certain time interval, or energy transfer time. The author uses this approach to describe spallation destruction of some metals.

\section{Experimental Data Analysis}

In the event of spallation loading, the compression pulse is created on one end of a sample as a result of interaction of the latter with a striker, accelerated by some means or other. The compression pulse, once passed on a sample, is reflected from a free end and returns into the sample in the form of tension wave under the influence of which the destruction occurs. Acting pressure pulse parameters are restored in terms of running speed of free surface of a sample and depend on the properties of striker materials, its speed and sizes.

The magnetic pulse technique makes possible to extend the potential of this pattern by means of formation of transient pressures under control within a wide range of parametric variation directly at the edge of the sample [8]. The technique is highly adjustable to different loading patterns, permitting to test samples with crack-type macro-defects in accordance with the loading scheme, illustrated in Fig. 2.

\subsection{Threshold Destruction Loading}

In $[25,26]$ one can find data for some rocks from testing, performed under spallation loading conditions, with the use of the magnetic pulse loading technique. 
Simple stress condition, occurring at spallation area under known parameters of the load pulse, allows us to calculate the parameter $\tau_{L}$, while taking into account (6).

In the event of testing samples with crack-type macro-defects, their results given in Fig. 3, the stress condition at the crack apex is largely predetermined by its design. The experiments, described above, were set with the use of standard samples, their general view given in Fig. 2. Numerical analysis of wave loading of samples, conducted in ANSYS [27] environment, consistently with actual sizes of pre-crack and loading pulse time parameters, shows that actual sizes of the fracture apex have immense influence on potential energy variation, traced at a maximum stress point. Concurrently, there can be observed a significant difference between calculation data for defects with actual sizes and those to be calculated while taking into account an approach described in [28] for a crack tip. In Fig. 6 are given the values of the parameter

$$
P E(t)=\int_{0}^{t} \varepsilon(s, x) \sigma(s, x) d s
$$

at a point $\mathbf{M}$ (see Fig. 2), which corresponds to maximum stresses.

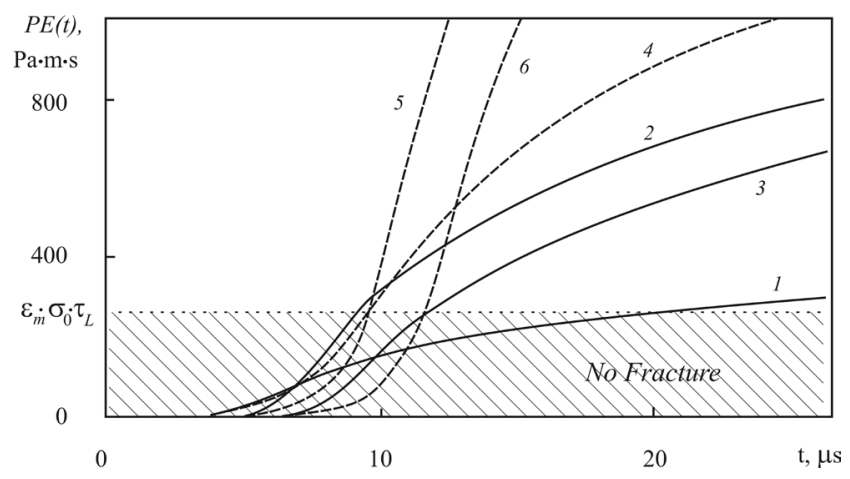

Figure 6. Dependence in PMMA sample at pre-crack length of: 1 and 4 $-0 \mathrm{~mm} ; 2$ and $5-1.5 \mathrm{~mm}, 3$ and $6-3 \mathrm{~mm}$, loaded by $320 \mathrm{MPa}$ pulse of 5.6 microsecond duration -curves 1-3 and of 11.6 microsecond duration.

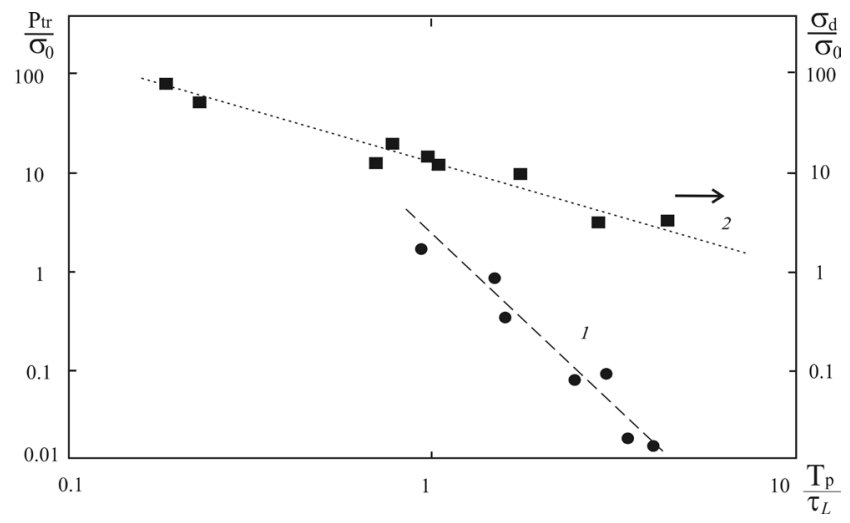

Figure 7. Generalized dependence for various materials of threshold destruction loading for testing samples with macro-defects -1 and along the spallation destruction pattern -2 .

Based on the analysis of numerical simulation of stressed state at the apex of the crack with actual sizes, it is possible both to describe the observed in [14] delay of fracture process $\tau_{d}$ of PMMA samples (Fig. 4), and to find the parameter $\tau_{L}$, its value coinciding with the one, based on the results of the spallation loading pattern sample testing, with an accuracy of $\pm 5 \%$. As one can conclude from the simulation results, represented in Fig. 6, the fulfillment of the condition (6) would occur at different moments of time, depending on the nature of the macro defect and loading duration.

Generalized material strength dependence on relative pulse duration, based on the data analysis, made with the use of the condition, described at (6), is given in Fig.7, curve 2. Basic mechanical properties of the materials, tested with application of the magnetic pulse technique, are given in Table 1.

Table 1. Properties of the materials, tested with application of the magnetic pulse technique.

\begin{tabular}{|c|c|c|c|c|c|c|}
\hline № & Material & $\begin{array}{c}\sigma_{0}, \\
\mathrm{MPa}\end{array}$ & $\begin{array}{c}\mathrm{c}_{1}, \\
\mathrm{~m} / \mathrm{s}\end{array}$ & $\begin{array}{c}\rho, \\
\mathrm{kg} / \mathrm{m}^{3}\end{array}$ & $\begin{array}{c}\mathrm{E}, \\
\mathrm{GPa}\end{array}$ & $\tau_{\mathrm{L}}, \mu \mathrm{s}$ \\
\hline 1 & Limestone & 12,4 & 3780 & 2570 & 48 & 1,75 \\
\hline 2 & Marble & 6,2 & 3790 & 2550 & 67 & 2,75 \\
\hline 3 & PMMA & 72 & 2450 & 1140 & 3,97 & 4,2 \\
\hline 4 & $\begin{array}{c}\text { Gabbro } \\
\text { diabase }\end{array}$ & 17,5 & 5600 & 3286 & 76 & 11 \\
\hline 5 & Granite & 5,2 & 4250 & 2670 & 35 & 12,4 \\
\hline 6 & Sandstone & 4,12 & 5100 & 2520 & 39 & 14,7 \\
\hline
\end{tabular}

Here $\sigma_{0}$ - static tensile strength, $c_{1}$ - longitudinal wave velocity, $\rho$ - density, $\mathrm{E}$ - Young`s modulus, $\tau_{\mathrm{L}}$ - energy accumulation time.

\subsection{Fracture energy at the pulse action}

Let us consider the behavior of the dependencies $L_{c r}(P)$, obtained for different materials in similar loading time modes, represented in Fig.3. In each case these dependencies are characterized by threshold behavior. When $P>P_{t r}$ the dependence of the propagated crack length on the amplitude of a pressure pulse with certain duration is expressed by:

$$
\left.L_{c r}(P) \approx \frac{d L_{C r}(P)}{d P}\right|_{P_{t r}} \cdot\left(P-P_{t r}\right)=k_{L P} \cdot\left(P-P_{t r}\right)
$$

Functional parameters, represented in Fig. $8 \mathrm{~A}={ }_{\mathrm{Cr}} / \tau_{L}$ и $B={ }^{k} L P / \tau_{L}$, can be explained as constant values $A=$ const $_{A}\left(\tau_{L}\right)$ and $B=$ const $_{B}\left(\tau_{L}\right)$ at a given value of exposure time.

The distinctive features were observed in loading mode with characteristic pulse duration of the order of 4 microseconds. Extra tests with other values of loading pulse duration were set for material №3 (see Fig. 8). The shorter the fracture pulse is, the steeper is the parameter $A\left(\tau_{L}\right)$ and the parameter $B\left(\tau_{L}\right)$, while the longer fracture pulse duration causes an opposite effect. Test points, 
corresponding to the fast loading mode, are marked as $3 f$, while those, corresponding to the slow mode, as - $3 s$.

The dependence

$$
C\left(\tau_{L}\right)=\left(\frac{d L_{c r}}{d P} \cdot P_{c r}\right)
$$

illustrates the lack of response to exposure time (Fig. 8). Functional parameter $C\left(\tau_{L}\right)$ can be explained as spatial scale of accumulation area of the energy, needed to initiate the destruction process (crack propagation start), or the critical zone.

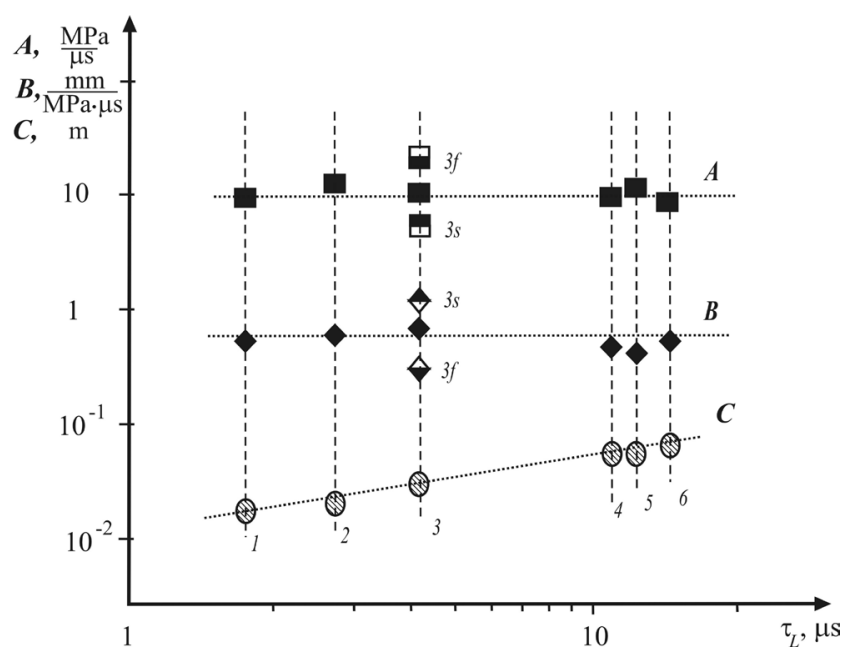

Figure 8. Fracture process parameters. Descriptors 1-6 correspond to materials, represented in the Table in respective rows.

All studied samples are characterized by efficient exposure to shock-wave loading and generic stress state, when values of critical threshold loading at the crack edge $P_{t r}$ and the stress value at the crack apex are related by parameter $k_{P \sigma}(T)$, which depends on loading duration.

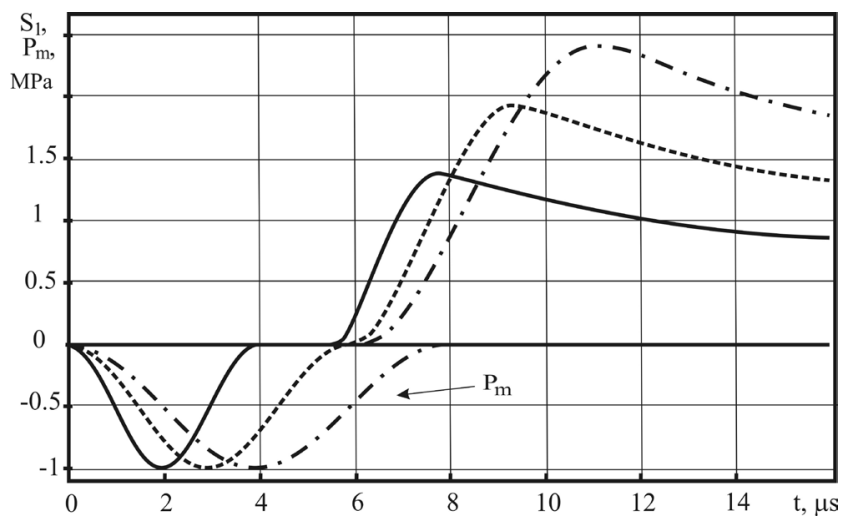

Figure 9. Dependence of pressure at the crack edge $P$ and stress value $S_{1}$ at the crack apex on time. At loading pulse duration 4 microseconds solid line, 6 microseconds - dashed line, 8 microseconds - dot and dash line.

In Fig. 9 are given the results of numerical simulation in ANSYS environment [27] of the stressed state at the crack apex, with account of various values of loading pulse duration at the pulse $1 \mathrm{MPa}$ amplitude.
Generic PMMA samples (see Fig. 2) show, that for a pulse with duration $T \sim 4$ microseconds, the parameter $k_{P \sigma}$ $=1.38$, and increases nearly linearly with the growth of loading pulse duration within the analyzed range (at $T=6$ microseconds: $k_{P \sigma}=1.92$; at $T=8$ microseconds: $k_{P \sigma}$ $=2.42$ ).

According to A.A. Griffith [29], for crack propagation the stored energy should suffice to produce new surfaces. The surface energy $\gamma$, released in process of elastic medium fracturing together with the occurrence of new surfaces, is related to the energy, stored in the medium, by expression

$$
\gamma=\pi \sigma^{2} l / E
$$

where $E$ - Young`s modulus, $l$ - crack length.

This expression, with the account of simulation results, can be written as

$$
\gamma(P)=\pi / E \cdot k_{P \sigma}^{2} P^{2} l
$$

Assuming the continuity of functions $\gamma\left(P_{c r}\right)$ and $P_{c r}\left(\tau_{L}\right)$, the derivative of surface energy can be expressed as

$$
\frac{d \gamma}{d P} \frac{d P}{d \tau_{L}}=\frac{\pi}{E} \cdot k_{P \sigma}^{2}\left(2 P \frac{d P}{d \tau_{L}} L_{c r}+P^{2} \frac{d L_{c r}}{d P} \frac{d P}{d \tau_{L}}\right)
$$

The first summand represents the surface energy change in process of crack propagation, the second - represents the energy, needed for this process, and accumulated in the area of crack propagation. At a point, corresponding to $P_{t r}$ the crack length is $L_{c r}(P)=0$, therefore, the first summand of expression (13) becomes zero. Thus, when critical value is achieved at the area, corresponding to maximum value of the criterion (6), the accumulated energy becomes sufficient for a crack to start. Dividing both sides of the expression (13) by $\tau_{L}$ we get

$$
\frac{1}{\tau_{L}} \frac{d \gamma}{d P} \frac{d P}{d \tau_{L}}=\frac{\pi}{E} \cdot k_{P \sigma}^{2} P^{2} \frac{d L_{c r}}{d P} \frac{d P}{d \tau_{L}} \frac{1}{\tau_{L}}
$$

With respect to experimentally revealed dependencies $\frac{P_{t r}}{\tau_{L}}=$ const $_{A}\left(\tau_{L}\right)=A$ и $\frac{k_{L P}}{\tau_{L}}=$ const $_{B}\left(\tau_{L}\right)=B$ we get expression:

$$
\frac{d \gamma}{d P}=\frac{\pi}{E} \cdot k_{P \sigma}^{2} \cdot A^{2} \cdot B \cdot \tau_{L}^{3}
$$

Upon integrating (15), the relation between the surface fracture energy, actualized at the crack starting moment, and the parameter of material $\tau_{L}$ is revealed:

$$
\left.\gamma\right|_{P_{t r}}=\frac{1}{4} \frac{\pi}{E} \cdot k_{P \sigma}^{2} \cdot A^{3} \cdot B \cdot \tau_{L}{ }^{4}+C_{\gamma}
$$

The obtained dependence, accurate to the constant of integration $C_{\gamma}$, can be seen in Fig. 10, and represents the increase of energy, needed for a crack to start, as related to the increase of $\tau_{L}$. Such behavior of the dependence 
$\left.\gamma\right|_{P_{t r}}\left(\tau_{L}\right)$ can also be related to the growth of critical zone, the scale of which $C\left(\tau_{L}\right)$ also grows together with the increase of energy accumulation time $\tau_{L}$.

Analysis of the relation (16), exemplified by material 3 (Fig. 8), shows, that the starting surface energy increases together with the increase of the loading rate.

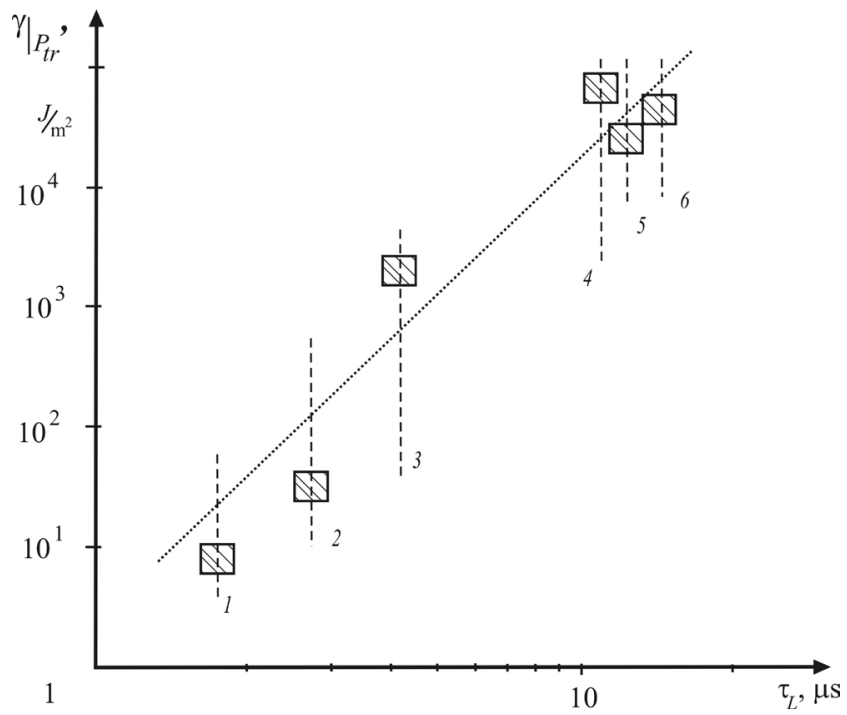

Figure 10. Dependence of the starting surface energy on $\tau_{L}$.

Results, obtained from analysis of experimental data for destruction of PMMA samples with crack-type macro defects under the action of pressure pulse with duration $T \sim 5.2 \mu \mathrm{s}$, demonstrate, that the starting rate of crack propagation $v_{0}$ is linearly related to the condition, when the pressure pulse amplitude $P_{c r}$ exceeds the threshold value $P_{t r}$ :

$$
v_{0} \approx K_{S} \cdot\left(P_{c r}-P_{t r}\right)
$$

where $K_{S} \approx 2,67 \cdot 10^{-6}$, dimensions of parameters given in SI system. Concurrently, the threshold amplitude of fracture loading $P_{t r}$ is determined with accuracy not less than $10 \%$ and equals $95 \mathrm{MPa}$. The fracture process was investigated by exposure to pulse action, formed by current pulse as the first half sinusoidal wave. In this mode it is possible to demonstrate, that the starting crack rate is related to maximum rate of change of pressure, applied to crack edges, by relation:

$$
v_{0} \approx K_{S} \cdot \frac{T}{2 \pi} \cdot\left(\left.\frac{d P_{c r}}{d t}\right|_{\max }-\left.\frac{d P_{t r}}{d t}\right|_{\max }\right)
$$

While the stresses are growing, the crack propagation starts at a certain moment $t^{*}$, that corresponds to violation of the condition (5). Differentiating (11) with respect to time, we get:

$$
\frac{d \gamma}{d t}=\pi l \frac{d}{d t}\left(\frac{\sigma^{2}}{E}\right)+\pi\left(\frac{\sigma^{2}}{E}\right) \frac{d l}{d t}
$$

At moment $t^{*}$ the crack length $l=0$ and the relation (19) takes the form:

$$
\frac{d \gamma}{d t}=\pi\left(\frac{\sigma^{2}}{E}\right) \frac{d l}{d t}=\pi\left(\frac{\sigma^{2}}{E}\right) v_{0} \equiv \pi(\sigma \cdot \varepsilon) v_{0}
$$

where $v_{0}$ - starting crack rate.

By integrating (20), it is possible to find how the surface fracture energy, released at the start of destruction, is related to the starting crack rate:

$$
\begin{aligned}
\left.\gamma\right|_{t} ^{*} & =v_{0} \int_{0}^{t} \varepsilon \cdot \sigma d s= \\
& =v_{0} \cdot \operatorname{PE}\left(t^{*}\right)=v_{0} \cdot\left(\varepsilon_{0} \cdot \sigma_{0} \cdot \tau_{L}\right)
\end{aligned}
$$

With respect to (17), the PMMA surface fracture energy increases together with the increase of the amplitude of pressure pulse, applied against the crack edges. For the starting rate of crack propagation $v_{0} \approx 570 \mathrm{~m} / \mathrm{s}$, that corresponds to the condition, when the pressure pulse amplitude exceeds the threshold value $\left(P_{c r}-P_{t r}\right) \approx 210$ $\mathrm{MPa}$, the surface fracture energy increases, compared to static value, by more than 30 times $\left(\gamma / \gamma_{0}=33\right)$.

This observation is valid for results of loading pulse testing at a given duration. Therefore, the dependency of the surface energy on the growth of loading rate would have similar character. The increase of the surface fracture energy at the growth of loading rate is observed in [30] and in experiments for metal destruction by spallation [31].

\section{Distinctive Features of Destruction during Shock-wave Loading}

The research for destruction of PMMA samples with crack-type macro defects and with uniform pressure pulse loading applied along the crack edges has revealed the existing dependency of the fracture energy on the starting rate of the crack.

If we relate this result to atomic and molecular bonds breaking, occurring as new surfaces emerge in the main crack area, then it seems rather surprising, since we can't expect the surge of atomic-binding energy at exposure time substantially higher than time of atomic-molecular system self-oscillations. Still, experiments, for instance [32-34], demonstrate, that the fracture process, independently on the material exposure technique, is conditioned not only by spatially localized propagation of the main crack (MC), but also by occurrence of multiple micro-discontinuities at the crack apex area (zone of maximum stresses), which influences the main crack propagation character. 
This assumption is also based on results of crack surface state analysis at different crack propagation rate values when exposed to loading pulse action. Thus, in [1] we demonstrate, that fracturing during main crack propagation is not one-dimensional, but is attended by occurrence of mesocracks, propagating angle-wise towards the main path (trajectory) to the depth of up to $100 \mu \mathrm{m}$, their inclination angle is loosely correlated with the crack propagation rate.

Formation of observed micro-discontinuities $[32,35]$ and mesocracks [1], propagating within the monitored velocity range $(100-600 \mathrm{~m} / \mathrm{s})$, requires more energy. Therefore, one can concede, that higher energy intensity, while new surfaces emerge, can be explained by a multi-level character of the destruction process, its localization not being determined solely by the main crack, occurring at macro-level.

Spatial scale of this process determines the dimensions of the area, where energy, necessary for the main crack to start, is being accumulated. When critical energy, introduced into this area, exceeds the threshold, defined by properties of this material within the time interval, or energy accumulation time, specific for this material, it leads to occurrence of dissipative processes and their development ends with the main crack formation.

As in order to elaborate the criterion we considered material properties, corresponding to complete failure of functionality, while these very properties (threshold strength, critical strain) can be established upon destruction of standard samples, this criteria approach is justified when describing destruction processes occurring at macro-level. However, as one can see in $[16,24,36]$, in order to analyze the scale hierarchy of the destruction, the thermodynamic approach can also prove to be efficient.

We demonstrate, that the use of the criterial approach to describe material fracture test data, obtained by application of different loading patterns, and based on estimation of the threshold potential energy, released in critical area during a specific time interval, allows us to reveal the relation between the principle fracture parameters and the energy accumulation time. This parameter can be regarded as a material property, being part of its basic characteristics, responsible for behavior of the mechanical system when exposed to pulse action.

Based on analysis of the development and application of pulse magnetic systems [37] and a loading device, represented by quasi-coaxial flat buses [21], significant increase of the amplitude (up to 5-7 GPa) of pressure, formed by magnetic pulse loading, seems technically justified. Concurrently, we foresee the perspective for testing metal samples with crack-type macro-defects, loaded in compliance with mode 1 .

\section{Acknowledgements}

Work is supported by Russian Federal Targeted Program for 2014-2020 Grant \#14.584.21.0019.

\section{REFERENCES}

[1] S. A. Atroshenko, S. I. Krivosheev, A. Y. Petrov. Crack propagation upon dynamic failure of polymethylmethacrylate, Technical Physics, Vol. 47(2), 194-199, 2002.

[2] S. A. Atroshenko, S. I. Krivosheev, Y. A. Petrov, A. A. Utkin, G. D. Fedorovskii. Investigation of fracture of spheroplastics under static and dynamic loading, Strength of Materials, Vol. 34(3), 273-276, 2002.

[3] S. I. Krivosheev. Experimental study of destruction of gabbro diabase under spalling loading condition, St. Petersburg State Polytechnical University Journal, No. 2, 115-117, 2003, ISSN 1994-2354, Russian.

[4] S. A. Novikov. Materials destruction under intense shock actions, Soros Science Education Jornal, No.8, 116-121, 1999, Russian.

[5] B. K. Barakhtin, Y. I. Mescheriakov, G. G. Savenkov. Statistical characteristics of the multiscale spallation of metal targets during dynamic loading and their relation to the mechanical properties of the materials, Technical Physics, Vol.55(1), 79-84, 2010.

[6] J. R. Klepachko, A. Braga. An experimental method for dynamic tensile testing of concrete by spalling, Int. Journal of Impact Engineering, No.25, 387-409, 2001.

[7] S. I. Krivosheev, N. F. Morozov, Y. V. Petrov, G. A. Shneerson. Problems of fracture of brittle bodies under pulse loading, Materials Science, Vol. 32(3), 286-295, 1996.

[8] S. I. Krivosheev. Pulsed magnetic technique of material testing under impulsive loading, Technical Physics, Vol. 50(3), 334$340,2005$.

[9] M. A. Sadovskiy. Selected Works: Geophysics and physics of the explosion, Ed. by V.V. Adushkin, Moskow, Science, 2004, 440 p, ISBN 5-02-032960-6.

[10] G. A. Shneerson. Fields and Transients in Superhigh Pulse Current Device, Nova Science Publishers, Inc, New York, $1997,560 \mathrm{p}$.

[11] K Ravi-Chandar, W. G. Knauss. An experimental investigation into dynamic fracture: 1.Crack initiation and arrest, International Journal of Fracture, Vol.25, 247-262, 1984.

[12] I. Smirnov, Y. Sudenkov. Crack propagation in PMMA plates under various loading conditions, In: 13th International Conference on Fracture 2013 (ICF 2013), 16 June 2013 through 21 June 2013, Code 104511, Beijing; China, Vol.4, 2696-2705.

[13] J. R. Klepachko, Y. V. Petrov, S .A. Atroshenko, P Chevrier, G. D. Fedorovsky, S. I. Krivosheev at al. Behavior of particle-filled polymer composite under static and dynamic loading, Engineering Fracture Mechanics, Vol.75(1), 136-152, 2007, DOI: 10.1016/j.engfracmech.2007.02.016.

[14] A. N. Berezkin, S. I. Krivosheev, Y. V. Petrov, A. A. Utkin. Effect of delayed crack nucleation under threshold pulse loading, Doklady Physics, Vol. 45(11), 617-619, 2000. 
[15] K. A. Rahmatulin, Y. I. Shemiakin, Y. A. Demianinov, A. V. Zviaghin. Strength and fracture under transient loads, Universitetskaya kniga, Logos, Moskow, 2008, 624 p., Russian.

[16] A. Y. Uchaev, V. T. Punin, N. I. Selchenkova, E. V. Kosheleva. Materials in extreme conditions, the critical phenomena and phase transfer in the dynamic destruction, In: Joint international topical meeting on mathematics \& computation and supercomputing in nuclear applications $(\mathrm{M} \& \mathrm{C}+\mathrm{SNA}$ 2007), Monterey, CA, 1-8, 2007.

[17] N. Morozov, Y. Petrov. Dynamics of Fracture. Springer Science \& Business Media, 2000, 98 p.

[18] S. N. Zhurkov. Issues of solid bodies' strength, Vestnik AN USSR, No.11, $78-82,1957$, Russian.

[19] Q. B. Zhang, J Zhao. A review of dynamic experimental techniques and mechanical behaviour of rock materials, Rock Mech Rock Eng, Vol. 47, 1411-1478, 2014, DOI: 10.1007/s00603-013-0463-y.

[20] A. S. Grigoriev, E. V. Shilko, V. A. Skripnyak, A. Y. Smolin, S. G. Psakhie, A. M. Bragov, A. K. Lomunov, L. A Igumnov. The numerical study of fracture and strength characteristics of heterogeneous brittle materials under dynamic loading (Conference Paper).In: International Conference on Physical Mesomechanics of Multilevel Systems 2014, Tomsk; Russian Federation; 3 September 2014 through 5 September 2014; Code 109441.-AIP Conference Proceedings: 2014, Vol.1623, 175-178.

[21] S. I. Krivosheev, N. V. Korovkin, V. K. Slastenko, S. G Magazinov. Destruction of brittle materials by microsecond pressure pulses at their formation by magnetic pulse method, International Journal of Mechanics, Vol.9, 293-299, 2015.

[22] J. H. Hagenguth. Volt-time areas of impulse sparkover, AIEE Trans, Vol.60, 803-810, 1941.

[23] M. Darveniza, A. E. Vlastos. The generalized integration method for predicting impulse volt-time characteristics for non- standard wave shapes - a theoretical basis, IEEE Transactions on Electrical Insulation, Vol.23(3), 373-381, 1988.

[24] D. E. Grady. Unifying role of dissipative action in the dynamic failure of solids, Journal of Applied Physics, Vol.117, 165905, 2015, DOI: $10.1063 / 1.4918603$.

[25] S. I. Krivosheev. Megagauss fields and strength of materials. LAP LAMBERT Academic Publishing, 2011, 152 p, ISBN 13: 978-3-8433-0864-9.

[26] S. I. Krivosheev, V. K. Slastenko. Features of destruction process under pulse action, St. Petersburg State Polytechnical
University Journal, Vol. 2, 228-234, 2012, Russian.

[27] ANSYS Academic Research Customer \#00420725 (from 2010), CompMechLab SPbSPU,

http://www.compmechlab.ru/

[28] C. C. Ma, L. B. Freund. The extent of the stress intensity factor field during crack growth under dynamic loading conditions, Journal of Applied Mechanics, Vol.53, 303-310, 1986.

[29] A. A. Griffith. The phenomenon of rupture and flow in solids, Philosophical Transactions of the Royal Society, Vol.A221, 163-198, 1921.

[30] K. Xia, W. Yao. Dynamic rock tests using split Hopkinson (Kolsky) bar system - A review, Journal of Rock Mechanics and Geotechnical Engineering, Vol.7, 27-59, 2015.

[31] G. G. Savenkov, A. A. Gruzdkov, B. K. Barakhtin, N. V. Lebedeva. Experimental determination of the effective surface energy density in dynamic problems of fracture mechanics, Technical Physics, Vol.58(2), 207-212, 2013.

[32] A. M. Leksowskij, B. L. Baskin. Some aspects of nucleation and evolution of microscopic and mesoscopic cracks and quasi-brittle fracture of homogeneous materials, Physics of the Solid State, Vol. 53(6), 2011, DOI:

10.1134/S1063783411060199.

[33] E. N. Brown, K. J. Ramos, D. M. Dattelbaum, B. J. Jensen at al. In situ and postmortem measures of damage in polymers at high strain-rate. In: Dynamic Behavior of Materials, Vol.1, Proceedings of the 2014 Annual Conference on Experimental and Applied Mechanics, Editors: Bo Song, Daniel Casem, Jamie Kimberley, 53-59, 2014, ISBN: 978-3-319-06994-4, ISSN: 2191-5644, DOI: 10.1007/978-3-319-06995-1_10.

[34] Yang Yang, Peng Zhiqiang, Chen Xingzhi, Guo Zhaoliang, Tang Tiegang, Hu Haibo at all. Spall behaviors of high purity copper under sweeping detonation, Materials Science \& Engineering, Vol.A65, 636-645, 2016.

[35] A. P. Jivkov. Structure of micro-crack population and damage evolution in quasi-brittle media, Theoretical and Applied Fracture Mechanics, 1-9, Vol.70, 2014.

[36] R. I. Il'kaev, V. T. Punin, A. Y. Uchaev, S. A. Novikov, E. V. Kosheleva, L. A. Platonova, N. I. Sel'chenkova, N. A. Yukina. Time features of the dynamic failure of metals attributable to the hierarchic properties of dissipative structures - cascade of failure centers, Doklady Physics, Vol.48, No.11, 627-632, 2003.

[37] G. A. Shneerson, M. I. Dolotenko, S. I. Krivosheev. Strong and super strong pulsed magnetic fields generation, Walter De Gruyter Incorp rated, 439 p., 2014, ISBN: 3110251914 , ISBN13: 9783110251913. 